\title{
MOTIVACIONES PARA ACCIONES DE RSE EN RESORTS EN BRASIL
}

\section{MOTIVATIONS FOR ACTIONS OF CSR IN RESORTS IN BRASIL}

Souza, Claudio Alexandre (Universidade Estadual do Oeste do Paraná-UNIOESTE, Brasil) *

Rodrigues Soares, Jakson Renner (Universidade de Coruña) ***

Sánchez Fernández, María Dolores (Universidade da Coruña) ${ }^{* * *}$

\section{RESUMEN}

El objetivo de este artículo es presentar un estudio sobre las motivaciones que impulsan a un resort a implementar acciones de responsabilidad social empresarial (RSE), teniendo como base las variables del entorno, los stakeholders y las dimensiones de la sostenibilidad. Este trabajo es el resultado de una investigación bibliográfica procedente de la revisión de artículos y bibliografía sobre los resorts y RSE, principalmente en lo que se refiere a sus respectivas motivaciones. Para la sistematización de este estudio se creó y se utilizó la Matriz de Análisis de la Responsabilidad Social Empresarial (MARSE) integrando las variables del entorno utilizadas en esta investigación. Los resultados evidencian motivaciones sistematizadas, pero estas se comportan de manera única debido a la singularidad del producto turístico resorts y a la amplitud de relaciones sociales que los mismos poseen con sus respectivos stakeholders. Tanto la MARSE, creada para este estudio, como las conclusiones obtenidas, sirven como referencia para la realización de futuros estudios empíricos acerca de las motivaciones de la implementación de la RSE en resorts. La MARSE puede ser considerada como una forma original de analizar el área RSE, debido a la posibilidad de sistematización.

Palabras claves: Motivación, RSE, Resorts, Sostenibilidad, Stakeholders. JEL: M14, L20, M20.

\begin{abstract}
The purpose of the article is to present a theoretical essay about the motivations that drive a resort the achievements actions of corporate social responsibility, based on environment variables, stakeholders and dimensions of sustainability. This theoretical essay is the result of bibliographic research in journals and bibliographies on resorts and corporate social responsibility, especially regarding their respective motivations. To systematize this test was created and used the Corporate Social Responsibility Analysis Matrix (MARSE) integrating the variables analyzed in this study. The theoretical results are taken by reference motivations already systematized, however they behave in a unique way in this study by the tourism product uniqueness resorts and the extent of social relations that they have towards their respective stakeholders. Both MARSE created for this study, as the conclusions serve as a reference for future specific and empirical studies on CSR motivations resorts. The MARSE
\end{abstract}

* Departamento de Ciencias Sociais, Avenida Tarquinio Joslin dos Santos, 1.300

Polo Universitario 85870-650 - Foz do Iguacu, PR - Brasil, 961. cas_tur@yahoo.com.br

** Rúa da Maestranza, 9, 15001 A Coruña .jakson.soares@udc.gal

*** Departamento de Análisis Económico y ADE, área Organización de Empresas, Campus de Elviña. 15071 -

A Coruña. mmsanchezf@udc.es

Recibido: Noviembre de 2016. Aceptado: Diciembre de 2016. 
can be considered as a unique way to carry out analyzes in the CSR area, it enables the systematic.

Key words: Motivation, CSR, resorts, Sustainability, Stakeholders. JEL: M14, L20, M20.

\section{INTRODUCCIÓN}

Comprender las motivaciones que llevan a una organización privada, como los resorts, a que adopten acciones de responsabilidad social empresarial (RSE) dirigidas a la sostenibilidad es vital para saber cómo gestionar adecuadamente estas actividades. Saber qué hacer en esta materia es el resultado de comprender las variables que motivan a los gestores, a las organizaciones y a sus respectivos stakeholders, en función del entorno y a las acciones de RSE con base en sus dimensiones.

Este trabajo presenta un estudio sobre las perspectivas mencionadas. Esta investigación busca servir de referencia para una comprensión más concreta sobre las relaciones sociales y sus idiosincrasias. El objetivo de este artículo es presentar un ensayo sobre las motivaciones que impulsan a un resort a implementar acciones de RSE, con base a las variables del entorno, los stakeholders y las dimensiones de la sostenibilidad.

Entre los motivos que justifican la realización de este estudio, cabe destacar: la experiencia profesional de 30 años del primer investigador con los resorts. Tales referencias contribuyeron a despertar el interés por los estudios en esta área, motivando todo ello, la búsqueda de comprensión científica sobre las variables motivacionales relativas a las acciones de RSE en los resorts. Comprender, con base en un estudio sistematizado, las motivaciones de implementar acciones de RSE en los resorts es una de las razones por las cuales estos investigadores buscaron desarrollar este estudio.

Esta investigación es resultado de una revisión bibliográfica en periódicos y fuentes bibliográficas sobre los resorts y la RSE, principalmente en lo concerniente a las motivaciones para implementar estas prácticas. Para la sistematización de este ensayo se creó la Matriz de Análisis de la Responsabilidad Social Empresarial (MARSE), integrando las variables analizadas en el estudio.

Los resultados toman como referencia las motivaciones ya sistematizadas, comportándose estas de forma singular en este estudio, debido a la particularidad del producto turístico, los resorts, y por la amplitud de relaciones sociales que los mismos poseen con sus stakeholders.

Tanto la MARSE, creada para este estudio, como las conclusiones obtenidas, sirven de referencia para futuros estudios puntuales y empíricos para investigar las motivaciones de RSE en los resorts. La MARSE puede ser considerada como una forma original de realizar un análisis en el área de RSE, debido a que permite la sistematización para su estudio.

El ensayo presenta teorías sobre motivación, ya abordadas en otros sectores de la economía, aplicadas en este trabajo a los resorts. Para la comprensión del estudio, el artículo está estructurado en cuatro bloques: introducción, desarrollo subdividido en dos apartados, los resorts y las motivaciones para adoptar la RSE, consideraciones finales y, por último, se incluyen las referencias bibliográficas. 


\section{REVISIÓN DE LA LITERATURA}

\subsection{Resorts: análisis de las relaciones sociales con los stakeholders}

El resort es un tipo de alojamiento que posee especificidades que lo hacen singular para una investigación académica. Existen escasas investigaciones dedicadas a estudiar los resorts (Brey, 2011; Souza, 2013). Sin embargo, por la cantidad y por la importancia de estos emprendimientos turísticos que ya existen, o que se están construyendo en Brasil, son importantes para los stakeholders (Sampaio, 2009). Esta investigación busca contribuir al conocimiento sobre los resorts realizando un estudio sobre la relación de las acciones de RSE entre los resorts y los stakeholders. Identificar quienes son los stakeholders y como se dan estas relaciones será fundamental para la comprensión de cómo afecta la territorialidad a partir de las acciones de RSE. Los resorts serán analizados bajo la perspectiva de organizaciones privadas caracterizadas por su entorno interno y externo (Aldrich y Pfeffer, 1976). Las partes con las cuales un resort se relaciona serán analizadas bajo la perspectiva de la Teoría de los stakeholders, siendo considerados stakeholders las partes que se relacionan e influencian, o son influenciadas por una empresa, en este caso por el resort (Freeman y McVea, 2000). Esta investigación se apoya en la clasificación de las partes interesadas, que ofrece la Teoría de los Stakeholders, debido a la claridad y la posibilidad de identificación de las relaciones existentes entre una organización y la sociedad (Freeman y McVea, 2000), teniendo en consideración las limitaciones que presenta esta teoría (Donaldson y Preston, 1995). Con base en estos estudios se analizarán en esta investigación cómo los stakeholders están presentes en las relaciones en una organización tipo resort y cómo interactúan.

FIGURA 1: STAKEHOLDERS

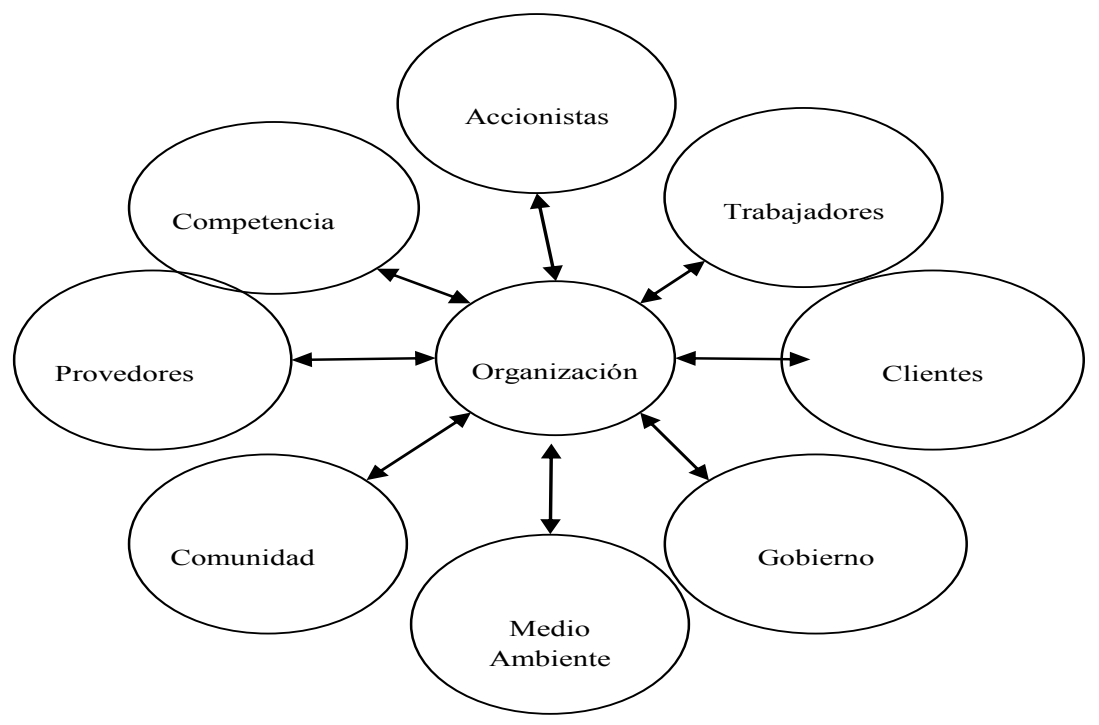

Fuente: Adaptado de Donaldson y Preston (1995, p. 69)

Este estudio enfoca el análisis de la RSE como una forma en que las empresas contribuyen al desarrollo sostenible. Las normas ABNT (2012) son guías que permiten 
implementar acciones de RSE y de sostenibilidad, estas normas correlacionan estas dos áreas, aunque existen estudios que muestran disconformidad en la unión entre la sostenibilidad y la RSE (Blackburn, 2007, p. 5) y la importancia de implementar acciones de RSE con el fin de obtener un desarrollo sostenible. Blackburn (2007) analiza en sus estudios las dimensiones de la sostenibilidad (ambiental, sociocultural y económica), identificando los aspectos internos y externos en una organización.

Las empresas estudiadas en este trabajo - los resort - serán analizadas con base en las relaciones con sus stakeholders en sus dos entornos (interno y externo), en todas las dimensiones de la sostenibilidad. Cada stakeholder puede tener diferentes relaciones e intereses en una organización (Romaniello y Amâncio, 2005), por lo tanto los stakeholders, en este estudio, serán distribuidos en consonancia al entorno. En esta investigación, los accionistas, los trabajadores y los clientes se encuentran localizados en el entorno interno de los resorts. El gobierno, la comunidad, el medio ambiente, la competencia y los proveedores se ubican en el entorno externo (Claro, Claro y Amancio, 2008). Los clientes, que en algunos estudios están ubicados en el entorno externo (Claro, Claro y Amancio, 2008), en este caso por la singularidad de un resort (OMT, 2001; Brey, 2011; Souza, 2013) en cuanto empresa privada del sector de turismo, se identifican en el entorno interno (Richard y Bacon, 2011; Souza, 2013). Esto se debe a que las empresas del sector de turismo, como los resorts, poseen una singularidad, la necesidad de que el cliente esté en el entorno interno o de producción para recibir el producto (OMT, 2001).

\begin{tabular}{|c|c|c|c|}
\hline \multicolumn{4}{|c|}{$\begin{array}{l}\text { CUADRO 1: MARSE - MATRIZ DE ANÁLISIS DE } \\
\text { LA RESPONSABILIDAD SOCIAL EMPRESARIAL }\end{array}$} \\
\hline & $\begin{array}{l}\text { Dimensión } \\
\text { Ambiental }\end{array}$ & $\begin{array}{c}\text { Dimensión } \\
\text { Sociocultural }\end{array}$ & $\begin{array}{l}\text { Dimensión } \\
\text { Económica }\end{array}$ \\
\hline Entorno Interno & \multicolumn{3}{|c|}{$\begin{array}{c}\text { Accionistas } \\
\text { Trabajadores } \\
\text { Clientes }\end{array}$} \\
\hline Entorno Externo & & $\begin{array}{c}\text { Gobierno } \\
\text { Comunidad } \\
\text { Medio ambiente } \\
\text { Proveedores } \\
\text { Concurrentes }\end{array}$ & \\
\hline
\end{tabular}

\subsection{EI Motivaciones para adoptar la Responsabilidad Social Empresarial}

¿Por qué una empresa que tiene como objetivo la riqueza inicia un proceso que no tiene asegurado un resultado financiero positivo? Ello requiere invertir recursos, cuyo gestor es el responsable, pero no propietario, existe una potencial posibilidad de que estos recursos retornen o se reinviertan en aspectos positivos para la organización. Los resorts, así como en otro tipo de empresas, están implementando acciones de RSE buscando los retornos financieros, la mejora de la imagen o, simplemente, las organizaciones realizan estas acciones porque ejercen presión los stakeholders para llevarlas a cabo. A pesar de lo comentado, hay que tener en consideración que existen algunos estudios que identifican motivaciones eminentemente altruistas (Font et al., 2012; Font, Garay y Jones, 2016). Enfocándonos en esta área de estudio, este texto está estructurado en tres partes en base al modelo propuesto 
resultado de una sistematización de las perspectivas identificadas en otras investigaciones sobre esta temática (Garay y Font, 2013; Font, Garay y Jones, 2016). La primera parte presenta las discusiones relativas a la teoría de la RSE basada en recursos, como la competitividad, la reducción de costes y la imagen de la empresa; seguidamente se expone, la discusión acerca de la legitimación de las acciones de los stakeholders, y se finaliza con los argumentos basados en razones altruistas para la realización de las acciones en materia de RSE por parte de los tomadores de decisiones.

\subsection{Teoría basada en recursos - TBR}

Se observa en las últimas décadas una evolución en lo relacionado con la percepción de la relación entre la RSE y la gestión de los recursos. En la década de 1990, Freeman (1994) y Jones (1995) argumentaron que el objetivo de la gestión es la búsqueda del beneficio financiero por parte de la empresa. Esta idea fue corroborada por Hillman y Keim (2001) como una relación consistente entre la gestión de los stakeholders y la generación de valor para los shareholders de la organización, una vez que estas tipologías de proyectos tienen baja posibilidad de ser copiados de forma efectiva por las otras empresas (Garriga y Mele, 2004). Porter y Kramer (2006), afirman que cuando la RSE es parte de la agenda de la empresa, puede llegar a minimizar o mitigar problemas inherentes a la Gobernanza Corporativa (Grant, 2016, p.39), por la inter-relación entre ambas. Todo ello contribuye a la competitividad de la empresa, cuestión identificada en el caso de los grupos de stakeholders clientes y trabajadores por Portney (2008) y Bonsón y Bednárová (2015). En esa situación es posible que los clientes sean susceptibles a comprar más, y también a pagar más por los productos de la empresa, que el personal trabaje de forma más eficaz y productiva (Porter y Kramer, 2006; Portney, 2008), o bien obtener otros efectos como favorecer la gestión de los recursos con la posibilidad de conseguir la reducción de problemáticas y la obtención de más recursos (Carroll y Shabana, 2010; Carroll, 2016). La forma de cómo se desarrolla la relación de los stakeholders se presenta como punto clave en la empresa para su propia gestión.

Los alojamientos hoteleros disponen de recursos naturales y humanos (trabajadores, clientes) (Bohdanowicz, 2005). Este hecho se aplica principalmente a los resorts, objeto de análisis en esta investigación. Otras características destacadas de los resorts es que disponen de: elevado número de unidades habitacionales (UH), necesitan de una gran área libre y considerable número de personal, lo que conlleva que sean emprendimientos turísticos de gran envergadura para un número considerable de clientes (Richard y Bacon, 2011; Brey, 2011; Souza, 2013). A todos los stakeholders les afectan las acciones de RSE de la empresa de forma directa o indirecta, obteniendo percepciones variadas y diferentes.

Las acciones basadas en recursos que están representadas en la MARSE, utilizada como referencia para análisis de los alojamientos tipo resorts, representan las relaciones sociales de los resorts con sus respectivos stakeholders. Consecuentemente, se debe tener en consideración la correlación entre las partes. Esta representación sirve para comprender la sostenibilidad y la teoría basada en recursos, siempre teniendo en consideración las interrelaciones de las acciones en otras dimensiones y entornos que no se comportan de forma estanca.

\subsection{Reducción de costes}

Las acciones de RSE debido al cambio de actitud que promueven en la gestión de la empresa, en relación a los recursos que esta utiliza, repercuten directamente en los costes de la empresa. La reducción de costes está restringida al entorno interno y la dimensión ambiental. 
Los investigadores Carrol y Shabana (2010) abordan la relación de las acciones de RSE desarrolladas dentro de la empresa -por los stakeholders accionistas que tienen repercusiones en los stakeholders clientes y personal-, entorno interno y, también, repercuten en las acciones que contribuyen a la reducción del uso de recursos, como por ejemplo, el consumo de agua y de energía eléctrica (Bohdanowicz, 2005; 2009), o que provocan actitudes que contribuyen a que esa reducción se produzca (Portney, 2008; Carroll y Shabana, 2010; Carroll, 2016), así como la formación específica para este fin (Bohdanowicz, 2006). El consumo está relacionado principalmente con los recursos de fuentes naturales, como el agua (Bohdanowicz, 2005; 2006; 2009), conectada directamente con la dimensión ambiental (Carroll y Shabana, 2010; Carroll, 2016). El resultado de estas acciones es percibido de forma directa por las empresas, ya que el consumo es medido mensualmente (Bohdanowicz, 2005; 2006; 2009; Souza y Costa, 2016). La reducción de estos recursos conlleva un impacto ambiental significativo, debido al gran consumo que tiene un alojamiento, principalmente del tipo resorts (Bohdanowicz, 2005; 2006; 2009). En el entorno ambiental interno se identifican como variables, en la matriz de análisis de la RSE (MARSE), con referencia a la reducción de costes las indicadas a continuación: mejora de la capacidad de gestión; acción de retorno de los stakeholders; cambio de postura de los trabajadores; reducción del uso de energía eléctrica; reducción del uso del agua; reducción de la generación de residuos y reducción de las bases energéticas. En la matriz se identifica que, gestionar los costes puede ser una forma de buscar el mismo objetivo para la corporación que para los stakeholders, mediante la aplicación de acciones responsables en las relaciones de gobernanza. Consecuentemente se toma una decisión que beneficia a la empresa, pero que también es positiva para los stakeholders, siendo ellos uno de los responsables para esta toma de decisión. Además de reducir el coste, es necesario saber cuánto exactamente se va a reducir y cómo se contribuye a ello.

\subsection{Imagen atractiva}

En general, las acciones de RSE son implementadas en las empresas por los cambios de perspectiva ante sus stakeholders, con el propósito de que las acciones resulten positivas para ellos. La imagen atractiva de la empresa está ligada a los entornos interno y externo y a la dimensión sociocultural. Los investigadores Garriga y Mele (2004), Carrol y Shabana (2010) y Carroll (2016) abordan la relación de las acciones de RS desarrolladas por los stakeholders y por los accionistas hacia todos los demás stakeholders, con el fin de promover la imagen de la empresa. Se identifican acciones enfocadas a este fin en el entorno interno (Portney, 2008), (McWilliams y Siegel, 2001, p. 120), para los clientes, mediante el desarrollo de proyectos ambientales, y para los trabajadores, aplicando una remuneración justa e implementando acciones formativas (Bohdanowicz, 2005; 2006; 2009; Garay y Font, 2011; Font, Garay y Jones, 2016). Se verifican también acciones en el entorno externo (Portney, 2008), principalmente en la dimensión sociocultural, mediante la implementación de proyectos en la esfera social (Bohdanowicz, 2009) y cultural (Garay y Font, 2011; Font, Garay y Jones, 2016). Las acciones que se desarrollan en la organización dirigidas a los diversos stakeholders buscan promover la imagen de la empresa, directa o indirectamente, para que tenga una repercusión positiva en todas las partes interesadas. 


\begin{tabular}{|c|c|c|c|}
\hline \multicolumn{4}{|c|}{$\begin{array}{c}\text { CUADRO 2: MATRIZ DE ANÁLISIS DE LA RSE - } \\
\text { MARSE (IMAGEN ATRACTIVA) }\end{array}$} \\
\hline ENTORNO & AMBIENTAL & SOCIOCULTURAL & ECONÓMICA \\
\hline INTERNO & ---- & $\begin{array}{l}\text { Retención de trabajadores; } \\
\text { Trabajadores satisfechos; } \\
\text { Atracción de trabajadores } \\
\text { cualificados; } \\
\text { Imagen de empresa confiable; } \\
\text { Imagen de empresa honesta; } \\
\text { Trabajadores proactivos; } \\
\text { Aumento de la productividad; } \\
\text { Clientes que toman decisiones } \\
\text { favorables. }\end{array}$ & ----- \\
\hline EXTERNO & $\begin{array}{l}\text { Reducción de la } \\
\text { emisión de } \\
\text { carbono; } \\
\text { Conservación de } \\
\text { la biodiversidad. }\end{array}$ & $\begin{array}{l}\text { Calidades asociadas a la honestidad } \\
\text { y a la confiabilidad; } \\
\text { Cadena de proveedores locales } \\
\text { confiables; } \\
\text { Posicionamiento diferenciado por las } \\
\text { acciones de difícil reproducción; } \\
\text { Necesidad de realizar adecuadas } \\
\text { acciones de marketing. }\end{array}$ & ---- \\
\hline
\end{tabular}

La imagen de la empresa, con referencia a la honestidad, la confiabilidad y la buena reputación, está vinculada directamente a las acciones que ella ejecuta, deliberadamente o no, tanto positiva como negativamente, y esto se refleja en la percepción de los stakeholders. Hillenbrand y Money (2007) identifican una fuerte relación entre la reputación de una empresa y su responsabilidad social. Es decir, cuanto más socialmente responsable sea la empresa, mejor es la reputación de la misma y, por otra parte, el desarrollo de acciones irresponsables que afecten a las partes interesadas influye negativamente en la reputación de la organización.

Se identifica incluso que, los stakeholders tienen noción de que reputación y responsabilidad son dos cuestiones diferentes, pero las relacionan como parte de un solo concepto. Es decir, a pesar de ser diferentes, la relación es directa. No basta tan sólo con realizar una acción de RSE, es tan fundamental desarrollar la acción como saber comunicarla al público idóneo, de la forma correcta. Y aun teniendo en consideración todos los aspectos mencionados no hay garantías de los efectos, los indicios y las posibilidades de obtener los resultados esperados, sin embargo hay que tener en consideración las partes interesadas son orgánicas en la relación e inter-relacionables entre sí.

Un alojamiento hotelero puede planificar y organizar una acción de RSE, pero no necesariamente prever y controlar sus resultados en los stakeholders, en función de las peculiaridades observadas en las relaciones entre las partes. Para ello, es necesario tener una gestión técnicamente correcta sobre las acciones de RSE con el fin de que se comprenda cómo efectivamente se reflejan en las relaciones sociales con los stakeholders afectados.

Las acciones de RSE exigen a las empresas que las ejecuten con una planificación adecuada, con el fin de que sepan cuáles son los posibles reflejos en las partes interesadas. De hecho, varios estudios destacan la necesidad de datos precisos y honestos, para saber implementarlos de forma correcta, cómo comunicarlos y percibir con qué stakeholders la acción desarrollada puede contribuir a una posible promoción positiva de la imagen 
competitiva pretendida por parte de la empresa (Bohdanowicz, 2005; El Dief y Font, 2010b; Font et al., 2012; Font; Garay y Jones, 2016). Además, hay que tener en consideración que, las acciones de RSE pueden contribuir a una mejora en el atractivo de la imagen cuando se realizan de forma voluntaria y altruista (El Dief y Font, 2010b), posicionando la imagen de la organización de forma diferenciada (Garay y Font, 2011; Font, Garay y Jones, 2016). Es decir, la motivación que impulsa a la empresa a realizar una acción puede ser derivada de la singularidad debido a la difícil reproducción, proporcionando beneficios duraderos en la imagen de la organización.

\subsection{Competitividad}

Diversos estudios analizan la competitividad de un resort con base en los resultados económicos y financieros y en función de la productividad que las acciones de RSE promueven en el área de cobertura de las empresas privadas. La competitividad está ligada al entorno externo de la dimensión económica. Los investigadores Garriga y Mele (2004), Porter y Kramer (2006) y Campbell (2007) abordan la relación de las acciones de RSE con el mercado -stakeholders- el gobierno, la comunidad, los proveedores y la competencia del entorno externo (Garriga y Mele, 2004), las posibles externalidades (Beltratti, 2005), la competencia (Hillman y Keim, 2001) y los proveedores (Campbell, 2007). Y principalmente esta relación puede estar vinculada con los proveedores locales (Font, 2008) y con los proveedores con algún tipo de acción considerada de orden ambiental (Garay y Font, 2011) y por consiguiente, trabajar de forma adecuada la RSE con estos stakeholders puede generar resultados positivos en las relaciones de orden financiera - dimensión económica (Carroll y Shabana, 2010; Carroll, 2016; Sydnor; Day y Adler, 2014).

A continuación se analizan los datos resultantes de investigaciones que abordaron acciones ambientales, socioculturales y económicas con relación a la RSE en alojamientos hoteleros, buscando identificar las motivaciones basadas en recursos que influyen en la implementación de las actividades aquí discutidas, y también se pretende descubrir si estas afectan a las partes interesadas. Se analizan los elementos que afectan a los recursos necesarios en las operaciones de este tipo de empresa, iniciando con cuestiones relativas a la competitividad, siguiendo con la reducción de costes y finalizando con la imagen atractiva. Las variables que conforman la matriz de análisis de la RSE, con referencia a la competitividad son: relación con las externalidades; ventaja social transformada en financiera; alineada con los objetivos de la empresa; mejora de las relaciones con los stakeholders e implementación de forma integrada y efectiva (Font, 2012).

La competitividad es uno de los elementos que motivan a muchas acciones empresariales en materia de RSE. Esto deriva en el interés suscitado por el potencial de alcance que tiene implementar esta tipología de acciones la empresa en las diferentes relaciones de las empresas con las distintas partes interesadas del entorno externo. Por lo tanto, es preciso conocer el entorno externo en que actúa la empresa y cómo las externalidades infieren en las partes interesadas de este entorno, para que las empresas puedan, en función de las relaciones que desarrollan, proporcionar los retornos que buscan para sí y para los stakeholders.

\subsection{Teoría de los Stakeholders}

Los stakeholders son las partes interesadas en las relaciones con los resorts que, por fuerza de esta relación tienen intereses en las acciones desarrolladas por la organización. Por 
ello, las acciones de RSE son asimiladas como una de las formas de gestionar estas relaciones. Desde la década de 1960, Davis (1960) y Bowen (1953 apud Carroll, 1999) ya afirmaban que otras partes están afectadas por las decisiones de los gestores, y que tendrían, por lo tanto, derecho a ser consultadas para la toma de decisiones. Algunas investigaciones afirman que existe un "contrato" implícito que aborda la necesidad de que la empresa actúe de forma "justa" con los stakeholders involucrados, motivado por obligaciones éticas en su área de cobertura (Freeman, 1994; Donaldson y Dunfee, 1994; 2000; 2002; Jeurissen, 2004). Se puede decir que académicamente, y con base en hechos empíricos de los estudios, desde la perspectiva del mercado se ha reconocido el derecho de los stakeholders hace más de medio siglo (Davis, 1960).

En las investigaciones de McWilliams, Siegel y Wright (2006) se identifica que las empresas están adoptando prácticas de RSE en función de la presión de los stakeholders. Sin embargo, también se adoptan prácticas de RSE como forma para alcanzar los objetivos de la organización (Garriga y Mele, 2004) y no solo por reconocer los derechos de los stakeholders. Tal vez por ello, se identifiquen fallos en los procesos de gestión de estas relaciones, como la falta de involucrar de manera efectiva a las partes interesadas (Adams y Zutshi, 2004). En algunos trabajos resaltan que, la forma de gestionar las acciones de RSE no se está realizando de forma adecuada (Freeman, 1994), por lo que se identifica la necesidad de invertir esta situación y la de organizar los stakeholders en las empresas, con el fin poder actuar como partes integradas en el entorno que están relacionados (Sternberg, 2010). Después de identificar que existe la posibilidad de obtener resultados positivos de estas relaciones, diversos estudios demuestran la necesidad de maximizarlos y, por tanto, actuar con las partes interesadas dentro de sus respectivas peculiaridades (Freeman, 1994; Garriga y Mele, 2004). Por razones meramente financieras, algunas empresas implementan acciones de RSE enfocadas a sus stakeholders, las cuales están motivadas únicamente por la búsqueda de la maximización de los resultados, y aun así algunas organizaciones no consiguen alcanzarla. Se verifica que, no se reconocen a los stakeholders como parte integrante del proceso, pero sí cómo parte necesaria para un fin específico.

Los stakeholders son el motivo para que la empresa implemente determinadas acciones, pero también deben ser partes interesadas y activas en el proceso, para que los resultados sean beneficiosos para todos los stakeholders. Incluso en este contexto de actuación de las empresas, se identifican dos atributos de los stakeholders en relación a la organización: algunos tienen poder de influenciar y otros tienen poder de reclamar; aunque no siempre están presentes los dos poderes (Mitchell, Agle y Wood, 1997). Es importante destacar que, los stakeholders actúan en consonancia con sus preocupaciones y su capacidad de comprensión de la situación (Marrewijk, 2003, p. 98). Y se espera que todos los stakeholders sean responsables de sus actos en todas las áreas de cobertura (Sternberg, 2010), tanto en relación a los cobros, como en las acciones de influencia, ya que el propio derecho de hacerlo es legítimo. El retorno que la firma le proporciona a aquellos grupos de interés que tienen el derecho legítimo de reclamar o influenciar es interpretado como una acción de legitimación del derecho de los stakeholders (Mitchell, Agle y Wood, 1997). Algunos estudios argumentan que dicha legitimación daría soporte a un posible beneficio de rentabilidad, permitiendo combinar los objetivos financieros con los sociales de la empresa, en función de la implicación "real" de los stakeholders (Carroll y Shabana, 2010). La consideración de la implementación de una acción de RSE, por parte de una compañía, enfocada a obtener legitimación por parte de los stakeholders produce un cambio en el enfoque del poder, afectando a la relación entre la empresa y los grupos de interés (Freeman y Velamuri, 2005). A pesar de que las acciones de RSE de los stakeholders sean reconocidas como legítimas, no son habitualmente desarrolladas en las organizaciones. Las acciones de RSE se están 
ejerciendo en las relaciones sociales, no como un reconocimiento de la legitimidad, pero sí como una forma de obtención de lo que desea la empresa y no de lo que quieren las partes interesadas.

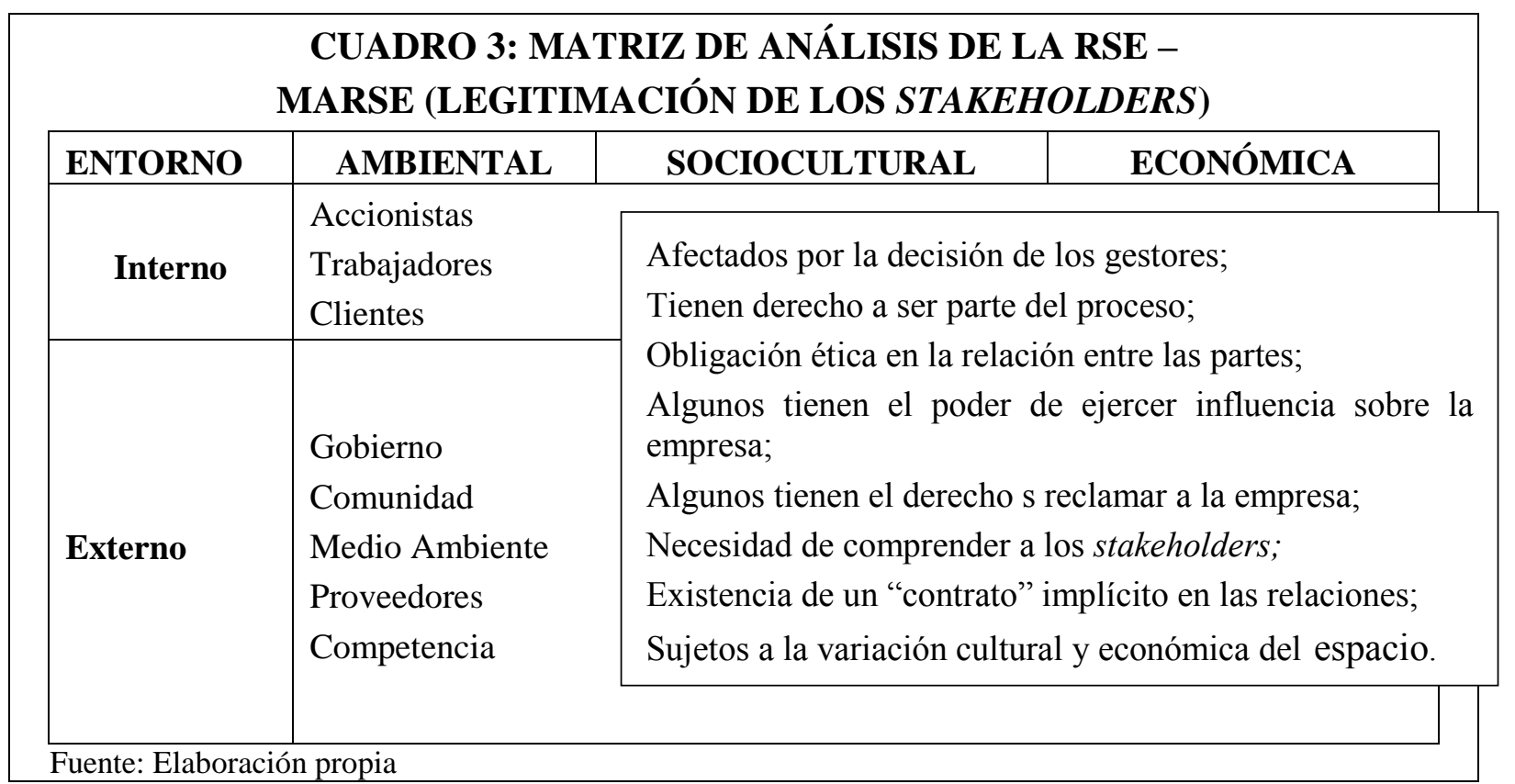

\subsection{Valores}

Las empresas son gestionadas por personas y muchas veces reflejan los valores de éstas en sus acciones, que se desarrollan enfocadas a los stakeholders. Una empresa puede ser gestionada implementando o no acciones de RSE, ya que para su efectiva realización se incurren en costes (Carroll y Shabana, 2010). Sin embargo, algunas empresas cuyos gestores participan en entidades que participan en proyectos sociales, desarrollan más acciones en el área de RSE (Campbell, 2007). Los aspectos filantrópicos, éticos y humanitarios son destacados como elementos básicos en las acciones de RSE (Carroll y Shabana, 2010). Las motivaciones altruistas son también identificadas como factores incentivadores de acciones de RSE, también existen razones de orden personal que impulsan a los gestores a invertir parte de los recursos de la empresa en el desarrollo de estas prácticas (Font y Garay, 2012; Font, Garay y Jones, 2016). Algunos estudios identifican motivaciones oriundas de elementos que forman parte del día a día particular de cada gestor, como catalizadores para las acciones de $\mathrm{RSE}$, los cuales inician o apoyan en las empresas en las que son responsables.

La donación de recursos, como acción de RSE, se realiza de varias maneras, en función de los diversos recursos gestionados por los alojamientos. Cuando se analizaron las prácticas altruistas de filantropía como elementos motivadores de acciones de RSE, se identificaron dos puntos: las donaciones y las acciones, originadas por razones eminentemente altruistas. Investigaciones sobre la donación de recursos financieros destinadas a proyectos apuntan que, más del 80 por 100 de los hoteles incluyen las donaciones en sus respectivos informes de RSE (Bohdanowicz y Zientara, 2008). Algunas empresas realizan donaciones de material para los stakeholders como resultado de acciones voluntarias (El Dief y Font, 2010b) y no como el resultado de presiones a los que pudieran estar sometidos, ya que algunos alojamientos hoteleros se involucran en proyectos de caridad (Bohdanowicz, Simanic y Martinac, 2005a). Esas acciones generan un posicionamiento positivo por parte de los stakeholders involucrados 
hacia los alojamientos hoteleros (Font et al., 2012). Algunas empresas sólo realizan estas donaciones porque a los gestores les interesa atraer una demanda futura (Ayuso, 2006). Otras investigaciones demuestran que existe una motivación originada por el deseo de practicar lo que denominaron "buenas acciones" (Bohdanowicz y Zientara, 2008; El Dief y Font, 2010a), lo que se caracteriza como razones altruistas por parte de los gestores de los alojamientos, aquellos que poseen gran formación y cualificación en el lugar de trabajo (Garay y Font, 2011; Font y Garay, 2012; Font, Garay y Jones, 2016). Se identificó que, el altruismo y la competitividad no son necesariamente excluyentes, y las acciones de RSE derivadas de estas motivaciones difícilmente son imitables (Garay y Font, 2011). De esta forma, tales relaciones pueden ser interpretadas como únicas en sus motivaciones y en su práctica, proporcionando a las partes involucradas momentos únicos que, influyen en las demás relaciones entre las partes interesadas, pudiendo en algunos casos generar resultados financieros, aspecto esperado por los gestores de los alojamientos.

\begin{tabular}{|c|c|c|c|}
\hline \multicolumn{4}{|c|}{ CUADRO 4: MATRIZ DE ANÁLISIS DE LA RSE - MARSE (VALORES) } \\
\hline Entorno & Ambiental & Sociocultural & Económica \\
\hline Interno & $\begin{array}{l}\text { Accionistas } \\
\text { Trabajadores }\end{array}$ & \multirow{2}{*}{\multicolumn{2}{|c|}{$\begin{array}{l}\text { Aspectos filantrópicos, éticos y humanitarios; } \\
\text { Decisiones de los gestores motivadas en razones } \\
\text { personales; } \\
\text { Acciones voluntarias que generan costes; } \\
\text { Gestores con experiencia en proyectos sociales; } \\
\text { Donaciones en más del } 80 \% \text { de los informes; } \\
\text { Difícilmente imitable. }\end{array}$}} \\
\hline Externo & $\begin{array}{l}\text { Gobierno } \\
\text { Comunidad } \\
\text { Medio Ambiente } \\
\text { Proveedores } \\
\text { Competencia } \\
\end{array}$ & & \\
\hline
\end{tabular}

El cuadro presenta, con objetivo didáctico, todas las motivaciones citadas en este apartado. Esta división es intencional, ya que en esta investigación fueron identificadas todas las motivaciones (Font et al., 2012) citadas, así como la respectiva subdivisión de la motivación basada en un recurso. Sin embargo, cabe resaltar que, esto no significa que todas las motivaciones se produzcan de forma parcial y en la misma proporción, tan sólo que todas están presentes en este trabajo. Para identificar cuál es la motivación que impulsa a los gestores a implementar prácticas de RSE, se tiene que realizar la investigación teniendo en cuenta la espacialidad y la temporalidad de este fenómeno (Bohdanowicz y Zientara, 2008; El Dief y Font, 2010a).

\section{METODOLOGÍA}

Los estudios de casos son trabajos que investigan fenómenos contemporáneos en profundidad y en su contexto real, especialmente cuando los límites entre el fenómeno y el contexto no son claramente evidentes (Yin, 2010). El multimétodo -cuando se tiene más de un caso- permite que se realicen análisis tanto caso por caso como de forma integrada, con base en las similitudes y en las diferencias de los casos múltiples investigados (Conejero y Nieves, 2007). Se recomienda la utilización de esta metodología en estudios con las siguientes condiciones: cuando se quiere responder el cómo y el porqué; cuando no se puede manipular el comportamiento de los objetos investigados; cuando se quieren cubrir ciertas condiciones 
consideradas fundamentales para entender el fenómeno investigado; y cuando no se tiene el límite claro del fenómeno y del contexto investigado (Yin, 2010; Pérez, 2016). Dicho método se aplica al estudio aquí descrito, ya que sus características se adecuan a esta propuesta metodológica.

La constitución de los casos seleccionados, para este estudio, puede ser descrita como muestra de conveniencia, dentro de los criterios determinados para selección de los mismos. Un estudio multimétodo es aplicable cuando utiliza varias fuentes de datos, tanto pueden provenir de evidencias cualitativas como cuantitativas (Yin, 2010, p. 41). Es decir, un estudio de caso puede incluir detalles e, incluso, ser limitado a evidencias cuantitativas (Yin, 2010, p. 41), no sólo cualitativa como comúnmente se presenta en ciertos estudios (Conejero y Nieves, 2007). Este estudio, como multimétodo, no posibilita una generalización estadística de los datos, tan solamente un análisis "generalizable", o generalización analítica, en que una teoría previamente desarrollada es utilizada como normalizada, lo que permite comparar los resultados empíricos del estudio de caso. Si dos o más casos demuestran apoyar la misma teoría, la réplica se puede afirmar (Yin, 2010, p.36 y p.61).

\subsection{Escalas}

Las escalas de Likert y de Guttmam serán utilizadas en este estudio con el objetivo de ampliar el potencial para la obtención de datos mediante la utilización del instrumento de investigación. Será utilizada la escala de Likert no comparativa, a nivel ordinal, con elección multivariada, con el objetivo de convertir en cuantitativo el instrumento utilizado, de forma que se pueda complementar la información que se busca. Utilizando el método mix to en esta investigación, las categorías y los ítems son los medios utilizados para la obtención tanto de datos cualitativos como cuantitativos, de forma simultánea, en el momento de la aplicación del instrumento para cada público-blanco investigado (Desselle, 2005). Los datos de orden cualitativo se obtuvieron a través del análisis directo de los ítems mediante la realización de entrevistas estructuradas y la observación in situ. En este caso se observó si el ítem existe y si identifica la evidencia de su realización. Los datos cuantitativos fueron obtenidos a través de la valoración de las categorías y de los ítems durante las entrevistas estructuradas y en la observación in situ. Estos datos fueron medidos en consonancia con las escalas presentadas para las categorías y para los ítems, y para cada uno de los públicos investigados. De esta forma, la escala posibilita una validación de los datos a través de la técnica denominada triangulación, mediante la recogida de evidencias y extensa observación de los hechos citados en la entrevista estructurada (Desselle, 2005). Con todo ello se busca identificar la existencia de datos empíricos, y comprender la relación orientada de las relaciones existentes entre las partes.

\subsection{Indicadores}

Se creó un indicador con el fin de poder valorar las acciones de RSE de los resorts (Crespo, 1996). El indicador de acciones de RSE posibilita valorar las acciones evaluadas para que se pueda analizar cuantitativamente, creando variables de análisis identificadas en la generación de las relaciones sociales entre los stakeholders, conforme a lo citado en esta metodología. El indicador tiene como base las acciones descritas en la norma de NBR 15401 y en la NBR 16001, de sostenibilidad para los alojamientos hoteleros y las acciones de responsabilidad social, respectivamente (ABNT, 2004; 2010; 2012). El indicador se presenta en formato porcentual (\%), lo que posibilita una mayor claridad en la lectura del resultado. Sin embargo, el resultado del indicador no solo aporta información de forma cuantitativa en 
las acciones de RSE realizadas por las organizaciones, sino que además involucra la regularidad, la efectividad y la validación de las acciones.

\subsection{Presentación y análisis de los datos}

La variable motivación para las acciones de RSE y su análisis son el resultado del estadístico tabulado de las variables observadas in situ en relación al conjunto de acciones analizadas. Esta motivación no se aplica de forma aislada a una u otra acción, ya que no fue el objetivo de este instrumento de investigación. Por lo tanto, se presentan en este trabajo los resultados representados en gráficos de sectores y de líneas. Y a partir de esta presentación, se realiza el análisis de los datos. Las variables sobre las motivaciones para implementar acciones de RSE se presentan en 4 momentos: el primero corresponde a una presentación general de las motivaciones en relación a todas las acciones de RSE investigadas; el segundo, se analizan la motivación de los stakeholders investigados; el tercer momento, son analizadas las motivaciones analizadas en función de los entornos de una organización y el cuarto, se presenta la motivación en consonancia a las acciones de RSE en base a las dimensiones de la sostenibilidad.

\section{GRÁFICO 1: MOTIVACIÓN PARA ADOPTAR ACCIONES DE RSE EN LAS RELACIONES EN LOS RESORTS (\%)}

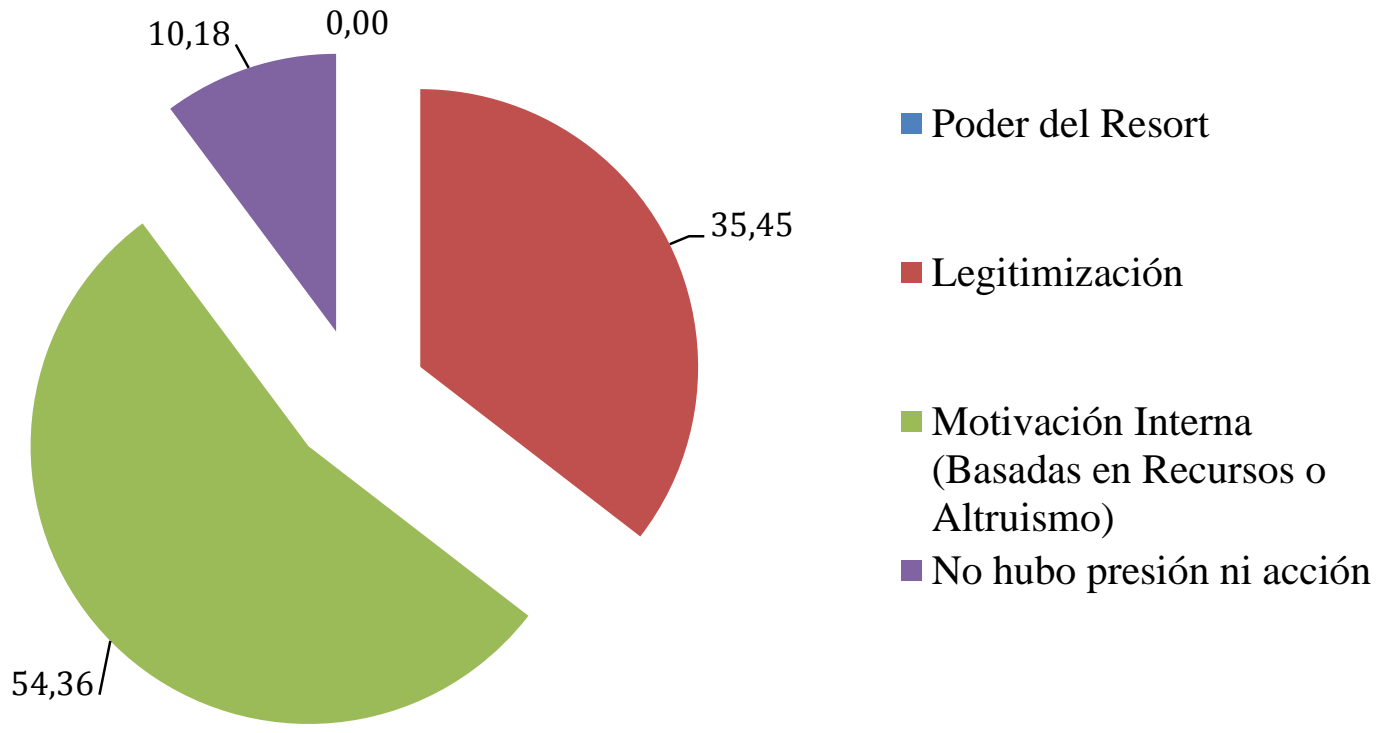

Fuente: Elaboración propia

Aproximadamente el 90 por 100 de las acciones de RSE en los resorts se realizaron en base o derivadas de motivaciones internas o como resultado para obtener mayor legitimación. Prácticamente el 55 por 100 de estas actividades se realizaron por motivos internos, en consonancia con el gráfico 1. Estas motivaciones basadas en recursos son las características identificadas en destacados estudios internacionales como las principales justificaciones para 
la implementación de acciones de RSE en alojamientos hoteleros (Bohdanowicz, 2005; 2006; Garay y Font, 2011), principalmente en resorts, debido a las características de los alojamientos de gran capacidad (Richard y Bacon, 2011; Brey, 2011). Se identificaron también como motivaciones internas, conforme el gráfico 1, razones altruistas, corroborando estudios anteriores (El Dief y Font, 2010; Garayy Font, 2011; 2013; Font, Garay y Jones, 2016). Formando parte de las motivaciones de las acciones de RSE en la empresa aproximadamente el 35 por 100- se encuentra la legitimación. Es un hecho tradicional que las acciones de RSE, combinándolas con la utilización de estrategias de comunicación, refuercen la legitimidad de los stakeholders (Davis, 1960; Bowen, 1953 apud Carrol, 1999; El Dief y Font, 2012). Comprender las efectivas razones que motivan las acciones de RSE de determinadas organizaciones privadas, en este caso los resorts, es un aspecto básico para que se puedan tomar decisiones considerando cómo se desarrollan las relaciones entre los stakeholders.

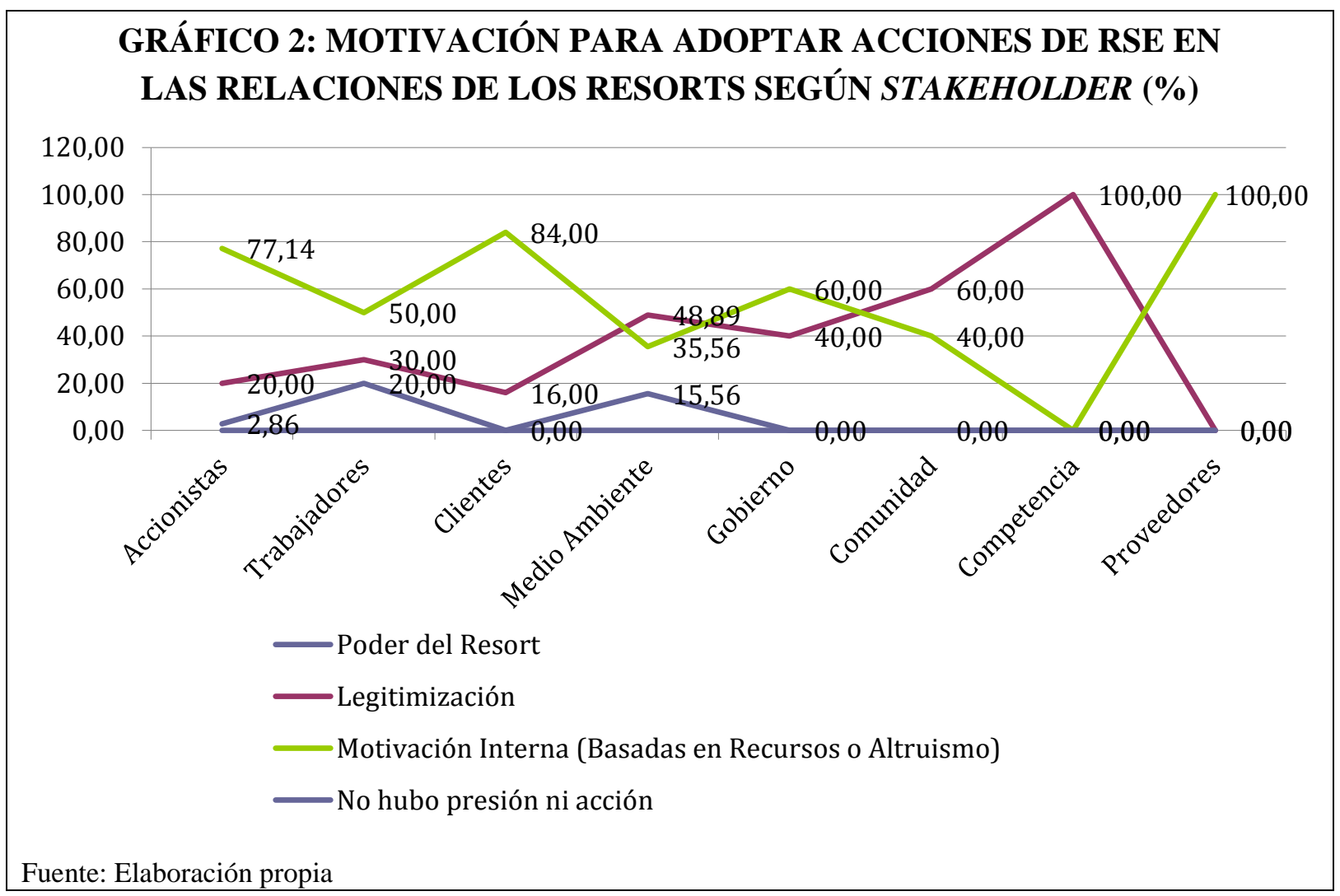

Las posturas adoptadas por las empresas, en cuanto a las acciones de RSE, son motivadas por diversas razones, y todas buscan alcanzar los objetivos que se propusieron (Adams y Zutshi, 2004; Garriga y Mele, 2004; Abaeiana, Yeoha, y Khong, 2014). Conforme a lo observado en el gráfico 2, en base a la legitimación, el 100 por 100 de las empresas están motivadas a desarrollar acciones de RSE. Se observó en este estudio que, las acciones identificadas son coordinadas por la Associação Brasileira de Resorts, para todos los resorts, y que estas empresas participen, a pesar de ofrecer resistencia ante ciertos proyectos, por entender que actuar con el stakeholder competencia es una forma de ayudar a maximizar los objetivos de su competencia (Freeman, 1994; Hillenbrand y Money, 2007; Garriga y Mele, 2004). Aceptar realizar estas acciones de RSE presupone el desarrollo de ciertas colaboraciones para alcanzar los objetivos de los resorts. Reconocer los demás resorts como 
competencia es admitir que son necesarios para alcanzar sus objetivos, aunque sea preciso actuar con él.

Se extrae del estudio que, es necesario para la obtención de mayor competitividad por parte de la empresa desarrollar acciones en colaboración con los proveedores, considerándolo como un stakeholder. En consonancia con el gráfico 2, la motivación interna corresponde al 100 por 100 de las acciones de RSE realizadas por los resorts, en relación con los proveedores. Estas prácticas se identifican como acciones que contribuyen a la búsqueda de un desempeño empresarial diferenciado (Jones, 1995; Garriga y Mele, 2004; Campbell, 2007). La búsqueda por la colaboración se produce principalmente en función de las relaciones desarrolladas por los proveedores locales (Bohdanowicz, 2006; Font, 2008). Se observó en este estudio, que existen relaciones de los resorts con los proveedores que desarrollan algún tipo de acción ambiental, social o cultural (Bohdanowicz, 2006; Garay y Font, 2011; Font, Garay y Jones, 2016). El stakeholder proveedor se interpreta en este estudio como parte del proceso para que el resort destaque competitivamente ante el mercado en que actúa.

El stakeholder cliente es el sujeto de acciones de RSE que, busca no sólo mejorar la imagen, sino también contribuir al aumento de la competitividad de los resorts (Garriga y Mele, 2004; Hillenbrand y Money, 2007; Carroll y Shabana, 2010). En el gráfico 3 se observa que las motivaciones internas corresponden al 84 por 100 de las acciones de RSE desarrolladas por los resorts, las cuales están orientadas hacia los clientes. Se resalta que, por las singularidades del producto turístico resort, los clientes se identifican tanto en el entorno interno como en el externo (Brey, 2011). Las acciones de RSE que se observan encaminadas a los stakeholders clientes, con base en la motivación interna identificada en este estudio, se encuadran tanto en el entorno interno (Hillenbrand y Money, 2007; Portney, 2008; McWilliams y Siegel, 2001, p. 120; Bohdanowicz, 2005; 2006; Garay y Font, 2011), como en el externo (Hillenbrand y Money, 2007; Portney, 2008; Garay y Font, 2011). Se resalta que, las acciones orientadas al stakeholder cliente pueden implementarse con el objetivo de gestionar emociones relacionadas con el producto recibido (Portney, 2008; Carroll y Shabana, 2010). Estas prácticas buscan presentar a este stakeholder la impresión de que se trata de un resort con servicios considerados confiables, honestos y de calidad (Garriga y Mele, 2004; McWilliams y Siegel, 2001). Comprender quiénes son los clientes y cómo las acciones de RSE desarrolladas les afectan es gestionar las relaciones entre las partes, con el fin de lograr comprender todo el potencial de interacción entre los stakeholders.

Las acciones del stakeholders accionista demuestran ser el resultado de la búsqueda para optimizar los recursos de la empresa que por ellos es gestionada. Con base en el gráfico 2, el 77,14 por 100 de las acciones de RSE desarrolladas por los accionistas son motivadas por razones internas. Los stakeholders accionistas, debido a la responsabilidad directa que poseen en la organización privada, en este caso los resorts, buscan realizar una gestión lo más ventajosa posible para la empresa (Beltratti, 2005; Sternberg, 2010). Una forma de actuar, bajo esta perspectiva, es a través de la implementación de acciones de RSE orientadas a todos los demás stakeholders, con los cuales las organizaciones tienen relaciones debido a intereses motivados por la reducción de costes, por la competitividad y por la imagen de la empresa (Garriga y Melé, 2004; Hillenbrand y Money, 2007; Carroll y Shabana, 2010). Se observó que, parte del 77,14 por 100 de las acciones de RSE implementadas en la empresa, se desarrollaron por motivaciones altruistas (El Dief y Font, 2010; Garay y Font, 2011; Font, Garay y Jones, 2016). Por parte del stakeholder accionista, las acciones las implementaron debido a motivaciones internas, y no necesariamente por reconocerlo como un derecho legítimo. 
Cuando se analizan los stakeholders gobierno y comunidad, se percibe un comportamiento inversamente proporcional, en el tocante a la motivación para desarrollar acciones de RSE en la empresa. Las prácticas implementadas en la empresa orientadas al stakeholder gobierno, están basadas al 60 por 100 en motivaciones internas (ver gráfico 2). Este resultado se deriva de la competitividad de las acciones que pueden proporcionar, para este stakeholder y no necesariamente por el reconocimiento del poder del stakeholder gobierno (Garriga y Mele, 2004; Porter y Kramer, 2006; Campbell, 2007; Garay y Font, 2011; Souza y Bahl, 2011). Por otra parte señalar que, las acciones de RSE realizadas por los resorts orientadas al stakeholder comunidad el 60 por 100 están motivadas para obtener, aumentar o mantener la legitimación de la organización. El 40 por 100 restante fue inversamente distribuido entre los dos stakeholders aquí tratados. Es decir, se reconoce el poder, los derechos y las actitudes del stakeholder comunidad en el 60 por 100 de las acciones orientadas para este stakeholder (Adams y Zutshi, 2004; Mcwilliams; Siegely Wright, 2006).

Los stakeholders trabajadores son objetivo del desarrollo de varias acciones de RSE, principalmente en función de los beneficios fiscales que pueda tener la organización, y no necesariamente que reviertan en el personal. Conforme al gráfico 2, se observa que el 50 por 100 de las acciones de RSE realizadas para los stakeholders trabajadores tuvieron como justificación motivaciones internas, y el 30 por 100 la legitimación. Algunos estudios apuntan que son comunes estas acciones en base a la motivación interna en las relaciones con los stakeholders trabajadores, ya que contribuyen en la reducción de costes (Campbell, 2007; Carroll y Shabana, 2010; Raub y Blunschi, 2014). Y más específicamente esta reducción de costes se observa en el caso de los alojamientos hoteleros, en los cuales se desarrollan acciones efectivas que reducen el consumo de energía eléctrica y de recursos hídricos (Bohdanowicz, 2005; 2006; Bohdanowicz y Martinac, 2007). Otra tipología son las acciones de RSE y que se implementan en la empresa a la cualificación del personal, la reducción de rotación, y a un desempeño más productivo y eficaz (Bohdanowicz, 2006; Portney, 2008). Por otra parte cabe señalar que el 30 por 100 de acciones de RSE están motivadas por la legitimación, como las acciones realizadas por el reconocimiento de las obligaciones legales y económicas, dentro del contexto jurídico laboral en Brasil (Font et al., 2012). Las acciones que desarrolla la empresa privada, orientadas a los trabajadores, son especialmente aquellas que están obligados a realizar por fuerza de ley. $\mathrm{O}$ bien otro motivo es porque proporcionan esta tipología de acciones un efectivo retorno. Las empresas no implementan estas actividades por entender que son necesarias o bien por los beneficios que generan para los stakeholders trabajadores.

El stakeholder medio ambiente, debido a las acciones jurídicas de la esfera ambiental y a la presión comercial, tiene acciones de RSE justificadas, tanto por legitimación como por motivaciones internas (Souza y Bahl, 2013; Souza y Costa, 2016). Este estudio identificó que aproximadamente el 50 por 100 de las acciones de RSE orientadas al stakeholder medio ambiente fueron motivadas por la legitimación, y aproximadamente el 35 por 100 fueron realizadas en base a la motivación interna. Muchas acciones de legitimación son el resultado de colaboraciones con Organizaciones No Gubernamentales (ONG), unidades de conservación y poder público, por reconocer sus demandas en cuestiones de orden ambiental (Souza y Bahl, 2013; Souza, 2013). Además cabe señalar que, ciertas acciones de RSE se implementan por el resultado positivo que posibilitan en el campo de mercadotecnia, no solo para los stakeholders involucrados directamente, sino también se benefician los restantes grupos, por el aspecto "comercial" que la utilización de estas imágenes posibilita para la competitividad (Ayuso, 2006). Es decir, se tiene en consideración la implementación de estas actividades no sólo debido a cuestiones de orden jurídico-administrativas, también se 
reconoce el valor de las acciones de RSE encaminadas al stakeholder medio ambiente, la competitividad en las organizaciones privadas.

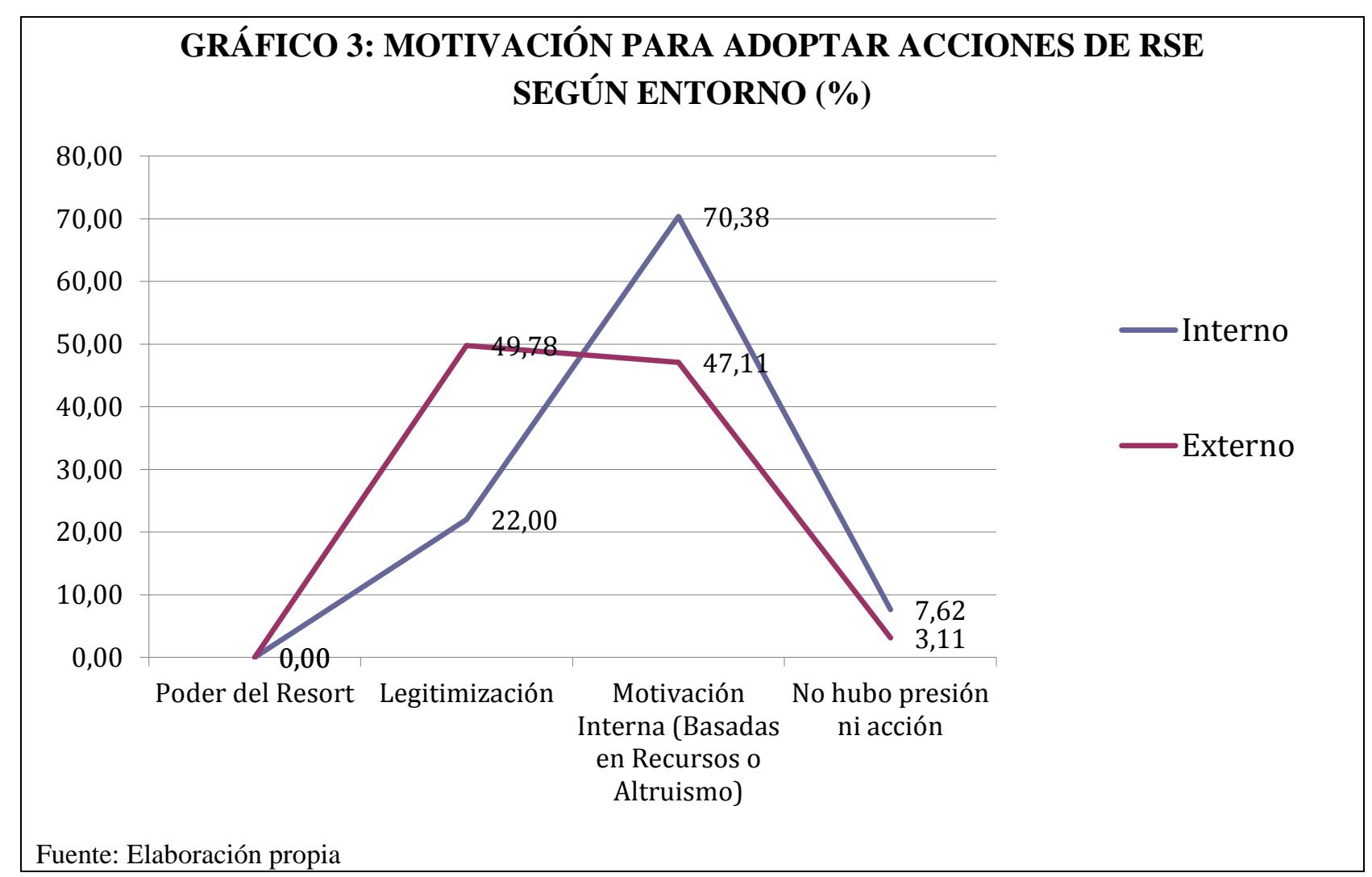

De los resultados se deduce que, mientras el entorno interno destaca por la motivación interna, el entorno externo demuestra un equilibrio entre la motivación interna y la legitimación, en el tocante a las razones que justifican las acciones de RSE que se implementan en la organización. La motivación interna corresponde al 70 por 100 de las acciones de RSE orientadas al entorno interno en consonancia con el gráfico 3. Cuestiones relativas principalmente a costes se justifican mediante la implementación de las acciones que afectan al entorno interno (Bohdanowicz, 2005; 2006; Portney, 2008; Carroll y Shabana, 2010). En cuanto al entorno externo, quedan divididas entre las acciones de RSE que se desarrollan en la empresa en base a justificaciones por cuestiones de costes, competitividad e imagen de la organización, como las realizadas por el reconocimiento de los derechos de posicionamiento de los stakeholders de ese entorno (Garriga y Mele, 2004). Quedan por investigar las acciones de forma individualizada, con el fin de poder comprender las respectivas motivaciones e influencias en las organizaciones privadas.

La dimensión económica de la sostenibilidad tiene aproximadamente el 80 por 100 de sus acciones justificadas con motivos internos. Los costes, la competitividad y la mejora de la imagen para obtener un mejor desempeño son las razones que alcanzan un 77,50 por 100 de las acciones de RSE identificadas en los resorts investigados. Las acciones encaminadas a la reducción de costes confirman otros estudios que identifican esta práctica como la principal motivación para realización de acciones de RSE, en varias organizaciones privadas, y en varios alojamientos hoteleros investigados (Ayuso, 2006; Campbell, 2007; Carroll y Shabana, 2010; Portney, 2008; Bohdanowicz, 2005; 2006). Las acciones de RSE implementadas están orientadas directamente en la reducción de costes, y en la contribución 
para que puedan atraer la competitividad de las organizaciones privadas (Garriga y Mele, 2004; Porter y Kramer, 2006; Campbell, 2007; Portney, 2008; Sternberg, 2010). Asimismo, el uso de la imagen de las acciones de RSE puede ser positivo en la reducción de costes relacionados a los recursos energéticos, en sintonía con las tendencias actuales de la gestión racional de recursos como el agua y la energía eléctrica (Bohdanowicz; Simanicy Martinac, 2005; Garay y Font, 2011; Font et al., 2012). Las acciones implementadas en esta dimensión se desarrollan no solo para alcanzar resultados inmediatos, sino también, en muchos casos, para obtener resultados a medio y a largo plazo (Ayuso, 2006).

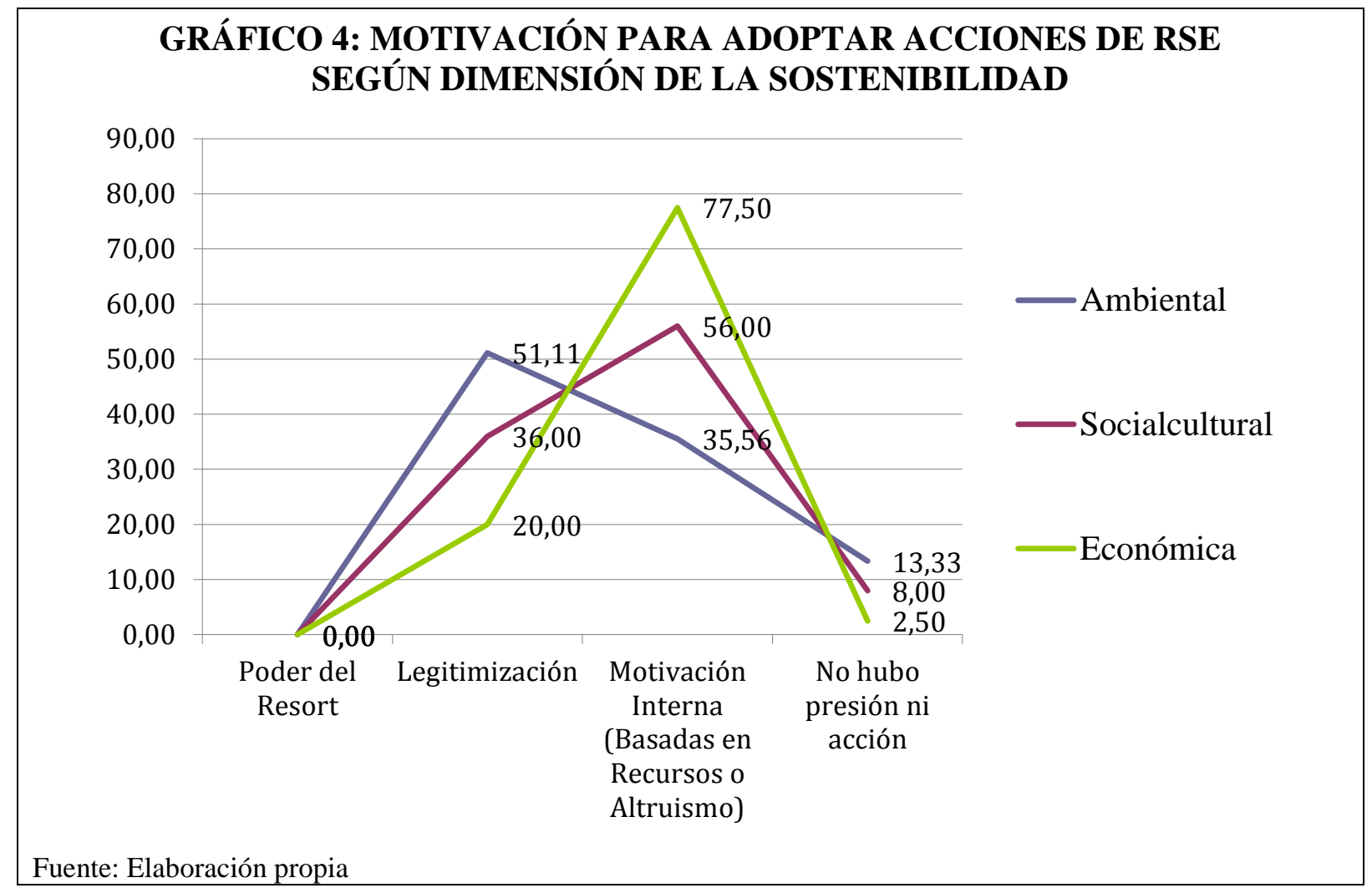

Las cuestiones de orden ambiental aún son asimiladas por las organizaciones como obligaciones, es decir, se llevan a cabo por el peso que se le da a las relaciones que se encuadran en esa dimensión. En este estudio, aproximadamente el 50 por 100 de las acciones de RSE implementadas por los resorts fueron motivadas por la legitimación, como por ejemplo, las que se orientan al stakeholder medio ambiente. En el 13,33 por 100 de las situaciones investigadas se implementaron acciones de RSE. Las acciones relativas al stakeholder medio ambiente son de tipo reactivo (Adams y Zutshi, 2004; McWilliams; Siegel y Wright, 2006; Sydnor, Day y Adler, 2014). El estudio identifica que, en la dimensión ambiental las acciones de RSE son efectivamente actividades de reacción en esta área, ante los escenarios que se presentan, en consonancia con los intereses de los resorts, o con el poder del grupo de interés involucrado.

En lo tocante a las acciones de RSE en la dimensión social se identificaron una cantidad considerable de acciones, directa o indirectamente, orientadas al stakeholder comunidad. Y de estas acciones solamente el 56 por 100 fueron implementados en base a motivos internos. A pesar de que diferentes estudios apuntan a que más del 80 por 100 de los alojamientos hoteleros efectúan donaciones de recursos financieros, como un tipo de acción en el campo 
social, los documentos analizados en este trabajo no mostraron esta práctica cómo una denominada acción de RSE (Bohdanowicz; Simanicy Martinac, 2005; Bohdanowicz y Zientara, 2009). Sin embargo cabe mencionar que se realizan donaciones y estas se desarrollan como colaboraciones con entidades de las comunidades del entorno de los resorts investigados (Bohdanowicz; Simanic y Martinac, 2005; El Dief y Font, 2012). Como se puede observar en el gráfico 4, el 36 por 100 de las acciones que conciernen a la dimensión social son de legitimación, y se producen como un ajuste en la conducta de los resorts (Adams y Zutshi, 2004; Mcwilliams; Siegel y Wright, 2006). Sin embargo, también se observó la práctica de acciones justificadas por motivos internos, las cuales se encuadrarían como actitudes altruistas, involucradas como parte de la política de los propietarios de algunos de los resorts investigados (Bohdanowicz y Zientara, 2008; El Dief y Font, 2010; Font, Garay y Jones, 2016). Se verifica que la dimensión social es el resultado tanto de acciones de RSE que pueden ser definidas como "buenas acciones", como de acciones que surgen en base a cimientos para la búsqueda de una competitividad fundamentada en diferenciales sociales (Garay y Font, 2011).

\section{CONSIDERACIONES FINALES}

Este trabajo busca comprender que motivaciones existen y pueden influenciar en la implementación o no de acciones de RSE en las empresas. Se verifica en esta investigación que muchas son las razones posibles que influyen en las empresas, cuando se implementa o se proyecta RSE en los alojamientos hoteleros.

En los últimos años las empresas están implementando prácticas de RSE de forma más intensa. No hay consenso entre los conceptos sobre el significado de estas actividades y las razones que motivan a las personas a realizarlas. Incluso no habiendo unanimidad en relación al concepto, los investigadores coinciden que estas actividades existen hace más de medio siglo y están en constante aumento. Sin embargo, no están suficientemente claros los motivos por los cuales se produce este incremento. Existen razones de orden personal motivadas por la postura de los gestores o por la búsqueda de la reducción de costes o, razones de orden corporativa, como la mejora de la imagen de la empresa, consciente de que estos factores posicionan a la organización de forma más competitiva.

¿Será la búsqueda de una posición competitiva lo que motiva a la empresa a implementar estas acciones? ¿O bien simplemente implementa estas prácticas para realizar lo correcto y consecuentemente estas acciones hacen que la empresa sea más competitiva? Hacer lo correcto es un principio de competitividad, aunque lo "correcto" no conlleve a que los resultados siempre se puedan alcanzar. Indiferentemente de uno u otro argumento, comprender los elementos que constituyen la RSE es básico para saber cómo actuar, qué realizar y cómo hacer para que las acciones sean rentables para todas las partes interesadas.

Diversos estudios apuntan que es necesario comprender los resultados de las acciones de RSE en los alojamientos hoteleros, y si éstos cambian en consonancia a las motivaciones que tienen los gestores para su realización. También es de interés averiguar cómo la implementación de esas acciones se refleja en los resultados y el modo en que ellas influyen. Hace falta comprender el comportamiento de cada una de las partes que componen las relaciones. Además, también es necesario conocer cuáles son las motivaciones que predominan. Conociendo la relación entre las motivaciones y los resultados, y como ellas actúan y pueden actuar en la gestión de los alojamientos hoteleros, sería posible alcanzar resultados adecuados para todos los stakeholders involucrados.

Una empresa necesita conocer e integrar las relaciones adecuadas con los stakeholders de la empresa. Un punto que se necesita investigar más sería conocer efectivamente quiénes 
son los stakeholders de una empresa en las relaciones que ella desarrolla, cómo identificarlos correctamente y cuáles son los intereses reales de las partes, con vistas a saber cómo gestionar de forma adecuada la relación con cada parte. Además también existe la necesidad de conocer exactamente quiénes son los grupos de stakeholders por empresa, ya que cada organización tiene un conjunto de características que la hace única y, por ello, tiene grupos de stakeholders únicos. Se debe priorizar la autonomía y la responsabilidad, en las relaciones con los stakeholders, no sólo por la responsabilidad de la empresa, sino también hay que tener en consideración todas las partes interesadas, dando autonomía y poder a las mismas y contribuyendo a una gestión diferenciada de la actual.

En cuanto a las variables que motivan la implementación de acciones de responsabilidad social empresarial de los resorts en Brasil, se puede percibir que no se comportan de manera homogénea. En lo relativo a las motivaciones, se observa que se manifiestan de forma diferenciada, en consonancia con el stakeholder y la acción de RSE que se analiza.

\section{BIBLIOGRAFÍA}

Abaeiana, V., Yeoha, K. K. y Khong, K. W. (2014): “An exploration of CSR initiatives undertaken by Malaysian hotels: Underlying motivations from a managerial perspective", Procedia - Social and Behavioral Sciences, n 144, pp. 423-432.

Adams, C. y Zutshi, A. (2004): "Corporates social responsibility: why business should act responsibly and be accountable", Australian Accounting Review, n 14(3), pp. 3-31.

Aldrich, H. E. y Pfeffer, J. (1976): "Environments of organizations author(s): reviewed work(s)", Annual Review of Sociology, no 2(1), pp. 79-105.

Ayuso, S. (2006): "Adoption of voluntary environmental tools for sustainable tourism: analysing the experience of Spanish hotels", Corporate Social Responsibility and Environmental Management, $\mathrm{n}^{\mathrm{o}}$ 13(4), pp. 207-220.

Ayuso, S. (2007): "Comparing voluntary policy instruments for sustainable tourism: the experience of the Spanish hotel sector", Journal of Sustainable Tourism, n ${ }^{\circ} 15(2)$, pp. 144-160.

Beltratti, A. (2005): "The complementarity betwen corporate governance and corporate social responsibility", The Geneva Papers, no 3(3), pp. 373-386.

Blackburn, W.R. (2007): The sustanability handbook: the complete management guide to achieving social, economics and environmental responsibility, Earthscan. London

Bohdanowicz, P. (2006): "Environmental awareness and initiatives in the Swedish and Polish hotel industries - survey results", Hospitality Management, no 25(1), pp. 662-682.

Bohdanowicz, P. y Martinac, I. (2007): "Determinants and benchmarking of resource consumption in hotels - case study of Hilton International and Scandic in Europe", Energy and Buildings, ${ }^{\circ}$ 39(1), pp. 82-95.

Bohdanowicz, P. y Zientara, P. (2009): "Hotel companies' contribution to improving the quality of life of local communities and the well-being of their employees", Tourism and Hospitality Research, ${ }^{\circ}$ 9(2), pp. 147-158.

Bohdanowicz, P. (2005): "European hoteliers' environmental attitudes: greening the business", Cornell Hotel and Restaurant Administration Quarterly, n 46, (2), pp. 188204.

Bohdanowicz, P., Simanic, B. y Martinac, I. (2005): "Environmental training and measures at scandic hotels, Sweden", Tourism Review International, $n^{\circ}$ 9(1), pp. 7-19. 
Bonsón, E. y Bednárová, M. (2015): "CSR reporting practices of Eurozone companies", Revista de Contabilidad - Spanish Accounting Review, n 18 (2), pp. 182-193.

Brey, E. T. (2011): “A taxonomy for resorts", Cornell Hospitality Quarterly, no 53(3), pp. 283-290.

Campbell, J. L. (2007): "Why would corporations behave in socially responsible ways? An institutional theory of corporate social responsibility", Academy of Management Review, no 32(3), pp. 946-967.

Carroll, A. B. y Shabana, K. M. (2010): "The business case for corporate social responsibility: a review of concepts, research and practice", International Journal of Management Reviews, $\mathrm{n}^{\circ}$ 12(1), pp. 85-105.

Carroll, A. B. (1991): "Corporate social responsibility evolution of a definitional construct". Business y Society, n 38(3), pp. 268-295.

Carroll, A. B. (2016): “Carroll's pyramid of CSR: taking another look", International Journal of Corporate Social Responsibility, n ${ }^{\mathrm{o}} 1, \mathrm{p} .3$.

Claro, P. B. O., Claro, D. P. y Amancio, R. (2008): "Entendendo o conceito de sustentabilidade nas Organizações", $R$. Adm., no 43(4), pp. 289-300.

Conejero, M. A. y Neves, M. F. (2007): “Gestão de créditos de carbono: um estudo multicasos", R.Adm., no 42(2), pp. 113-127.

Davis, K. (1960): “Can business afford to ignore corporate social responsibilities?", California Management Review, $\mathrm{n}^{\circ}$ 2(3), pp. 70-76.

Desselle, S. P. (2005): "Construction, implementation, and analysis of summated rating attitude scales", American Journal of Pharmaceutical Education, $n^{\circ}$ 69(5), pp. 1-11.

Donaldson, T. y Dunfee, T. W. (1965): "Ties that bind in business ethics: social contracts and why they matter", Journal of Banking y Finance, no 26(1), pp. 1853-1865.

Donaldson, T. y Dunfee, T. W. (1994): "Toward a unified conception of business ethics: integrative social contracts theory", Academy of Management Review, no 19(2), pp. 252-284.

Donaldson, T. y Dunfee, T. W. (2000): "Précis for: ties that bind", Business and Society Review, $\mathrm{n}^{\circ}$ 105(4), pp. 436-443.

Donaldson, T. y Preston, L. E. (1995): "The stakeholder theory of the corporation: concepts, evidence, and implications", Academy of Management Review, $\mathrm{n}^{\circ}$. 20(1), pp. 65-91.

El Dief M. y Font, X. (2010): “The determinants of hotels' marketing managers' green marketing behavior", Journal of Sustainable Tourism, n 18(2), pp. 157-174.

El Dief M. y Font, X. (2012): "Determinants of environmental management in the red sea hotels: personal and organizational values and contextual variables", Journal of Hospitality y Tourism Research, $\mathrm{n}^{\circ}$ 36(1), pp. 115-137.

Font, X. (2008): "Sostenibilidad y alivio de la pobreza en países en vías de desarrollo: el papel del hotelero e del investigador", Estudios y Perspectivas em Turismo, $\mathrm{n}^{\circ}$ 17(1), pp. 7-28.

Font, X. et al. (2012): "Corporate social responsibility: The disclosure-performance gap", Tourism Management, $\mathrm{n}^{\mathrm{o}}$ 33(6), pp. 1544-1553.

Font, X.; Garay, L.; Jones, S. (2016): "Sustaunability motivations and practices in small tourism enterprises in European protected áreas", Journal of Cleaner production, $\mathrm{n}^{\mathrm{o}}$ 137(20), pp. 1439-1448. 
Freeman, R. E. y McVea, J. (2001): “A stakeholder approach to strategic management”. In.: Hitt, M., Freeman, E. y Harrison, J. (Orgs.). Handbook of strategic management, Ed. Blackwell Publishing. Oxford

Freeman, R. E. y Velamuri, S. R. (2005): “A new approach to CSR: company stakeholder responsibility". Recuperado en 14 de Agosto, 2016, de: http://ssrn.com/abstract=1186223.

Freeman, R. E. (1994): "The politics of stakeholder theory: some future directions", Business Ethics Quarterly, n 4(4), pp. 409-421.

Garay, L. y Font, X. (2011): "Doing good to do well? Corporate social responsibility reasons, practices and impacts in small and médium accommodation enterprises", International Journal of Hospitality Management, $\mathrm{n}^{\circ}$ 31(1), pp. 329-337.

Garay, L. y Font, X. (2013): "Responsible behavior's, motivations, practices and results in small and médium tourism enterprises", Review of Corporate Social Responsibility, $\mathrm{n}^{\circ}$ 13(1), pp. 1-30.

Garriga, E.y Mele, D. (2004): "Corporate social responsibility theories: mapping the territory", Journal of Business Ethics, no 53(1), pp. 51-71.

Grant, R. M. (2016): Contemporary Strategy Analysis (9th Ed), Ed. John Wiley y Sons Inc, LTD. Chichester

Hillenbrand, C. y Money, K. (2007): "Corporate responsibility and corporate reputation: two separate concepts or two sides of the same coin?", Corporate Reputation Review, $\mathrm{n}^{\circ}$ 10(4), pp. 261-277.

Hillman, A. J. y Keim, G. D. (2001): "Shareholder value, stakeholder management, and social issues: what's the bottom line?", Strategic Management Journal, no 22(2), pp. 125139.

Jeurissen, R. (2004): “Institutional conditions of corporate citizenship", Journal of Business Ethics, no 53(1), pp. 87-96.

Jones, T. M. (1995): "Instrumental stakeholder theory: a synthesis of ethics and economics", The Academy of Management Review, $\mathrm{n}^{\circ}$ 20(2), pp. 404-437.

Marrewijk, M. (2003): "Concepts and definitions of csr and corporate sustainability: between agency and communion", Journal of Business Ethics, n'. 44(2), pp. 95-105.

McWilliams, A., Siegel, D. S. y Wright, P. M. (2006): "Corporate social responsibility: strategic implications", Journal of Management Studies, $\mathrm{n}^{\circ}$ 43(1), pp. 1-18.

Mitchell, R. K., Agle, B. R. y Wood, D. J. (1997): "Toward a theory of stakeholder identification and salience: defining the principle of who and what really counts", The Academy of Management Review, $\mathrm{n}^{\mathbf{0}}$ 22(4), pp. 853-886.

OMT. (2001): Introducción al turismo, OMT, Madrid

Pérez Hoyos, O. I. (2016): Metodología para la elaboración de estudios de caso en responsabilidad social, Universidad Externado de Colombia, Colombia.

Porter, M. E. y Kramer, M. R. (2007): "'Strategy and society: the link between competitive advantage and corporate social responsibility"., Harvard Business Review, no 84(12), pp. 78-92.

Portney, P. R. (2008): "The (not so) new corporate social responsibility: an empirical perspective", Review of Environmental Economics and Policy, $\mathrm{n}^{\circ} 2(2)$, pp. 261-275. 
Radhakrishnana, S. Chitraob, P. y Nagendra, A. (2014): "Corporate Social Responsibility (CSR) in Market Driven Environment”, Procedia Economics and Finance , n ${ }^{\circ} 11$, pp. 68-75.

Raub, S., Blunschi. S. (2014): "The Power of Meaningful Work: How Awareness of CSR Initiatives Fosters Task Significance and Positive Work Outcomes in Service Employees", Cornell Hospitality Quarterly, n 55(1), pp. 10-18.

Richard, S. y Bacon, P. (2011): Resorts: a foco on value, Ed. HVS, Madrid

Romaniello, M. M. y Amâncio, R. (2005): "Gestão estratégica e a responsabilidade social empresarial: um estudo sobre a percepção dos estudantes do curso de administração", REAd, $\mathrm{n}^{\mathrm{o}} 11(3)$, pp. 1-21.

Sampaio, C. F. (2009): O turismo e a territorialização dos resorts: a praia do Porto das Dunas como enclave em Aquiraz-CE, Dissertação de mestrado acadêmico em geografia, Universidade Estadual do Ceará, Fortaleza, Ceará, Brasil.

Souza, C. A. (2013): "Resort - Uma Análise da Discussão Conceitual e Histórica", Revista Hospitalidade, $\mathrm{n}^{\circ} 10(2)$, pp. 350-362.

Souza, C. A. y Bahl, M. (2013): "O poder nas relações dos resorts e o estado - caso de resorts com ações no MPF (Brasil)", Pasos, n 11(4), pp. 547-558.

Souza, F.M., Costa, H.A. (2016): "Razões que levam micro e pequenas empresas de ecoturismo ao comportamento responsável na Chapada dos Veadeiros (GO)", Revista Brasileira de Ecoturismo, no. 1(9), pp. 93-111.

Sternberg E. (2009): "Corporate social responsibility and corporate governance", Economic Affairs, $\mathrm{n}^{\circ}$ 2(4), pp. 5-10.

Sydnor, S, Day, J. y Adler, H. (2014): "Creating Competitive Advantage and Building Capital through Corporate Social Responsibility: An Exploratory Study of Hospitality Industry Practices", Management and Organizational Studies, $\mathrm{n}^{\mathrm{o}} 1$ (1), pp. 52-62.

Yin, R. K. (2010): Estudo de caso: planejamento e métodos, (4.ed), Ed. Bookman, Porto Alegre. 